\title{
Investigation of Self-assembly Structure and Properties of a Novel Designed Lego-type Peptide with Double Amphiphilic Surfaces
}

\author{
Liang Wang and Xiao-jun Zhao ${ }^{\dagger, *}$ \\ West China Hospital Nanomedicine Laboratory, Center for Regenerative Medicine and Institute for Nanobiomedical \\ Technology and Membrane Biology, West China Hospital, Sichuan University, Chengdu 610065, Sichuan, China \\ ${ }^{\dagger}$ Center for Biomedical Engineering, NE47-379, Massachusetts Institute of Technology, Cambridge, MA 02139-4307, USA \\ ${ }^{*}$ E-mail: xiaojunzhao08@gmail.com \\ Received July 22, 2010, Accepted October 19, 2010
}

\begin{abstract}
A typically designed 'Peptide Lego' has two distinct surfaces: a hydrophilic side that contains the complete charge distribution and a hydrophobic side. In this article, we describe the fabrication of a unique lego-type peptide with the AEAEYAKAK sequence. The novel peptide with double amphiphilic surfaces is different from typical peptides due to special arrangement of the residues. The results of CD, FT-IR, AFM and DLS demonstrate that the peptide with the random coil characteristic was able to form stable nanostructures that were mediated by non-covalent interactions in an aqueous solution. The data further indicated that despite its different structure, the peptide was able to undergo self-assembly similar to a typical peptide. In addition, the use of hydrophobic pyrene as a model allowed the peptide to provide a new type of potential nanomaterial for drug delivery. These efforts collectively open up a new direction in the fabrication of nanomaterials that are more perfect and versatile.
\end{abstract}

Key Words: Self-assembly structure and properties, Lego-type peptide, Amphiphilic surfaces, Pyrene, Fluorescence

\section{Introduction}

The self-assembly phenomenon, which is ubiquitous in nature, is the result of biological evolution. A large number of biologically functional macromolecules and organisms are accomplished through self-assembly. The development of self-assembling peptides is quickly becoming the most emerging nanoscale field and has gained rapid pace in recent years. It has prompted numerous studies of peptides such as lego-type peptides, peptide surfactants and peptide ink. ${ }^{1-3}$ 'Peptide Lego' forms well-ordered nanofiber scaffolds for 3D cell culture and for regenerative medicine. 'Peptide surfactants' are used for drug, protein and gene delivery as well as for solubilizing and stabilizing membrane proteins. 'Peptide ink' plays an important role in surface biological engineering. ${ }^{4-9}$

In this work, we focus on the molecular-designed 'Peptide Lego', discovered from a segment in a left-handed Z-DNA binding protein in yeast, which can be programmed for self-assembly in well-formed structures. ${ }^{10}$ Typical sequences of artificially fabricated lego-type peptides show regular repeats of alternating hydrophobic and hydrophilic residues along the complete sequence of the peptide, and the hydrophilic residues possess alternating positive and negative amino acids. This special arrangement of the residues gives the peptides two distinct surfaces: a hydrophilic side with the complete charge distribution and a hydrophobic side. ${ }^{11}$ These peptides exhibit the characteristic of beta-sheet structures and self-assemble into three-dimensional nanofiber scaffolds in water. ${ }^{12-15}$

In this project, we have followed traditional ideas for the fabrication of designer peptides to fabricate an amphiphilic peptide with a unique architecture. Our novel lego-type peptide consisted of 9 amino acids and was designed with two amphi- philic surfaces. With regard to the sequence of the peptide, alanine was designed as the hydrophobic backbone whereas Glu and Lys formed the hydrophilic district that provided ionic bonds in regular repeats. We designed the peptide with the Tyr amino acid and without the full-sequence ionic complements between the repeats of Ala-Glu and Ala-Lys. The introduction of Tyr mediated the charge residues distributed on both sides of the backbone to form a molecule with two amphiphilic surfaces, which is different from a typical molecule. In addition, the aromatic interactions may play a key role in the formation of nanostructures because they contribute free energy of formation, order and directionality to the self-assembly process. ${ }^{16}$ To detect its ability to spontaneously assemble into well-ordered nanostructures, we used CD, FT-IR AFM and DLS to investigate the self-assembly properties and behavior of the novel nanomaterial. As the results indicate, the novel peptide was able to undergo self-assembly and form a stable nanostructure in an aqueous solution. In addition, the investigation of the hydrophobic district demonstrated that the novel designed peptide was able to provide a new type of nanomaterial for delivering hydrophobic drugs. These efforts collectively open up a new direction in the fabrication of novel amphiphilic self-assembly peptides.

\section{Experimental}

Preparation of the peptide agent. The original peptide (sequence: AEAEYAKAK; theoretical mass 980.02) used in our study was commercially synthesized and purified ( $>95 \%$ ) by Shanghai Bootech Bioscience \& Technology Co. Ltd. Working solutions of $0.5 \mathrm{mg} / \mathrm{mL}$ were prepared with sterile water $(18 \mathrm{M} \Omega$; Millipore Milli-Q system) and stored at $4{ }^{\circ} \mathrm{C}$. The schematic 
(A)

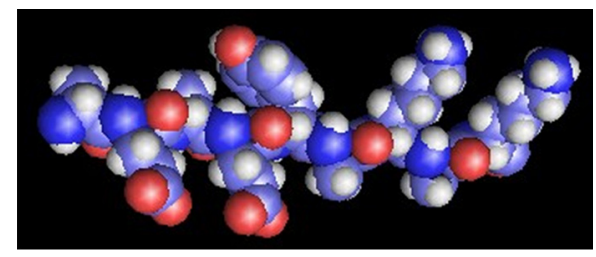

(B)

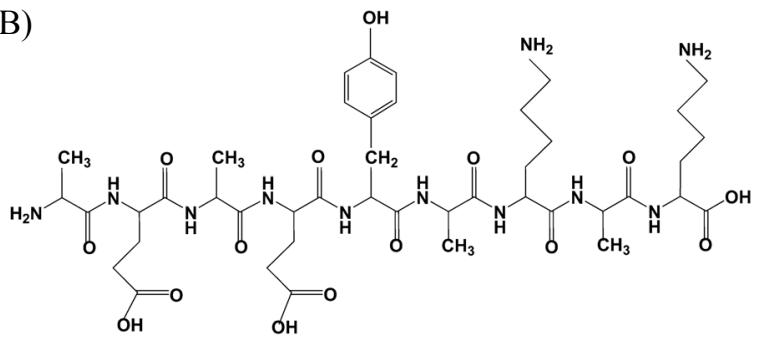

Figure 1. (A) Schematic molecular model of the lego-type peptide. The carbon atoms are shown in cyan, oxygen atoms in red, nitrogen atoms in blue, and hydrogen atoms in gray. The peptide is approximately $3.1 \mathrm{~nm}$ in length and $1.1 \mathrm{~nm}$ in width. (B) Chemical structure of the peptide.

molecular model, which is shown in Figure 1A, measured approximately $3.1 \mathrm{~nm}$ in length.

Circular dichroism (CD) spectroscopy. To detect the second structure of the peptide, the far-ultraviolet CD spectrum between $190 \mathrm{~nm}$ and $260 \mathrm{~nm}$ was collected using the wavelength scan model on a Model 400 Circular Dichroism Spectrophotometer (Aviv Biomedical, Inc.) at $25^{\circ} \mathrm{C}$. A $700-u L$ sample with a concentration of $0.2 \mathrm{mg} / \mathrm{mL}$ was used in a $2 \mathrm{~mm}$ path-length quartz $\mathrm{CD}$ cuvette. For each sample, the spectra were collected three times and analyzed to obtain an average.

Atomic force microscopy (AFM). Approximately $5 \mathrm{uL}$ of peptide working solution was deposited on the freshly cleaved surface of mica at room temperature. The surface was washed with an aliquot of $100 \mathrm{uL}$ of Milli-Q water to remove the unattached peptide and then air-dried. After air-drying, the AFM imaging approach was performed using an SPI4000 Probe Station and SPA-400 SPM Unit (Seiko Instruments, Chiba, Japan) using the dynamic force mode. The cantilever's free resonance frequency was approximately $125 \mathrm{KHz}$. AFM images were collected at scales of $5 \times 5 \mu \mathrm{m}^{2}$ and $10 \times 10 \mu \mathrm{m}^{2}$ with $512 \times 512$ pixel resolution to observe the nanostructure formed by the peptide.

Fourier transform infrared (FT-IR). The $10-\mathrm{mg} / \mathrm{mL}$ peptide solution was deposited on a crystal slide of zinc selenide and air-dried at room temperature. The IR spectrum was collected between wavelengths of 1500 and 2000 using a Spectrum One FT-IR spectrometer (Perkin Elmer).

Dynamic light scattering (DLS). A HORIBA LB-550 DLS nano-analyzer was used to measure the size distributions of the nanostructures. Approximately $1 \mathrm{~mL}$ of the peptide solution was added into a disposable sizing cuvette and kept at equilibrium at $25^{\circ} \mathrm{C}$ for $2 \mathrm{~min}$ prior to measurement. For each sample, data were collected five times and a final calculated average value was obtained.

Fluorescence measurement. Fluorescence spectrum measurements were performed at room temperature using a Hitachi
F7000 spectrophotometer with a stirring accessory. All samples were excited at $336 \mathrm{~nm}$ and the emission spectra were collected from $350 \mathrm{~nm}$ to $440 \mathrm{~nm}$ with the following parameters: excitation and emission slit width $=2.5 \mathrm{~nm}$; scan speed $=240 \mathrm{~nm} / \mathrm{min}$; response time $=0.1 \mathrm{~s}$. The pyrene crystals were prepared using tetrahydrofuran solution to obtain a pyrene concentration of $2.5 \mathrm{mM}$. A sample mixture of PY-THF ( $2 \mathrm{uL}, 2.5 \mathrm{mM})$ and peptide solution ( $500 \mathrm{uL}, 0.5 \mathrm{mg} / \mathrm{mL}$ ) was used for the fluorescence spectrum measurements, and a solution mixture of PY-THF (2 uL, $2.5 \mathrm{mM}$ ) and Milli-Q water (500 uL) was used as a control. Both samples were deemed to be stable when the fluorescence spectrum showed no change over a 72 -h period. ${ }^{17}$

\section{Results and Discussion}

Molecular model of the peptide. The typical lego-type peptide belongs to the group of amphiphilic peptides that form well-ordered nanofibers. These peptides contain two distinctive sides: one hydrophobic and the other hydrophilic. They form ionic bonds with regular repeats on the hydrophilic surface, e.g., EAKA-I, II. ${ }^{18}$ In this work, we designed a novel lego-type peptide that improved upon the EAKA model. The peptide consisted of 9 amino acids with the AEAEYAKAK sequence. The chemical structure of this type of lego-type peptide is shown in Figure 1B. Alanine formed the hydrophobic backbone in the peptide. The aromatic interactions, which contribute free energy of formation, order and directionality to the self-assembly process, may play a key role in the formation of nanostructures. ${ }^{16}$ Hence, we designed the peptide with the Tyr aromatic amino acid located between the repeat residues of Ala-Glu and Ala-Lys without the full-sequence ionic complementariness to form different ionic bonds on the different sides. Finally, we fabricated a novel lego-type peptide with both sides hydrophobic and hydrophilic.

Secondary structure properties of the peptide. It is well-known that the secondary structure plays an essential role in the selfassembly process of the peptide. $\mathrm{CD}$ has become increasingly recognized as a valuable structural technique for obtaining reliable data. As the CD spectrum data shown in Figure 2A indicates, the ellipticities showed a minimum near $195 \mathrm{~nm}$, corresponding to the characteristics of the random coil. However, it did not image the typical spectrum for an irregular secondary structure. ${ }^{19}$ The spectrum also indicated that our peptide was different from the traditional full-sequence complementary peptide, forming typical beta-sheet secondary structures.

Furthermore, we examined the nature of the secondary structure of the self-assembling peptide using FT-IR spectroscopy (Figure 2B). The IR spectrum of the peptide representing amide band I showed a vibration peak at $1628 \mathrm{~cm}^{-1}$. The exact frequency of the amide I absorptions proved the existence of a strong hydrogen bond and indicated that the lego-type peptide monomers were able to interact with each other and self-assemble in the solution to form the secondary structure through hydrophobic interactions and hydrogen bonds. ${ }^{20-21}$

Self-assembly behaviors investigated using AFM. The AFM system has evolved into a useful tool for direct measurement of micro-structural parameters and unraveling of the intermolecular forces at the nanoscale level with atomic-resolution cha- 

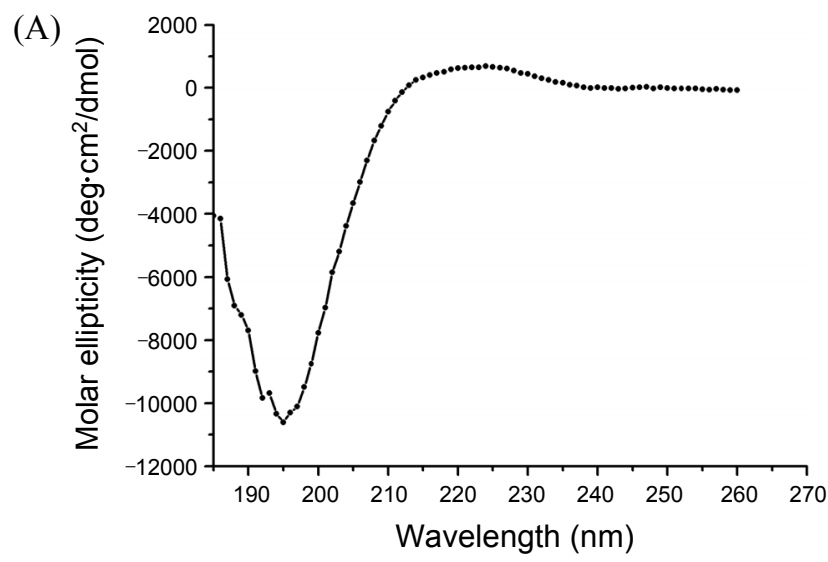

(B)

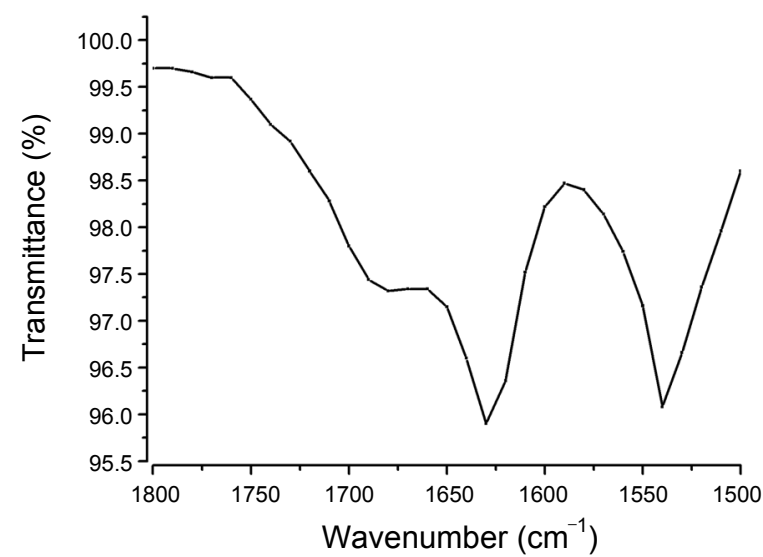

Figure 2. (A) CD spectra of the peptide $(0.2 \mathrm{mg} / \mathrm{mL})$ at $25{ }^{\circ} \mathrm{C}$ in the Milli-Q water. The spectra show a minimum near $195 \mathrm{~nm}$, which corresponds to the characteristics of the irregular secondary structure. (B) FT-IR spectra of the peptide. The absorption of amide I occurs at $1628 \mathrm{~cm}^{-1}$, which indicates that the peptide is involved in the formation of strong intermolecular hydrogen bonds and in hydrophobic interactions.

racterization. ${ }^{22}$ In this work, we used the AFM approach to gain more insight into the molecular organization of the self-assembling structures of the peptide (Figure 3). The properties of the peptide were studied using a peptide concentration of $0.5 \mathrm{mg} /$ $\mathrm{mL}$. The typical AFM morphological images of nanofibers were collected at diverse length. The images showed ordered and directional fibers in a dense arrangement instead of a meshwork. This arrangement may be due to the role of the aromatic Tyr residue in the fiber assembly. It is known that the lego-type peptide with beta-sheet structure has the ability to self-assemble into well-ordered nanofibers that further associate to form nanofiber scaffolds. This classification is based on the alternating positive and negative charge residues that are collected on the hydrophilic surface. However, the appearance of nanofiber tapes associated by our peptide monomers indicates that our novel peptide underwent a different self-assembly process. We speculate that the location of the charge residues on both sides of the peptide chain may also play an important role in the structure of the self-assembled product.

Detemination of nanostructures. In this work, DLS has been used to investigate the polymorphological size distributions of the nanostructure. We determined that the self-assembly of the
(A)

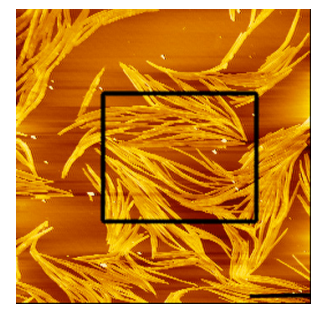

(B)

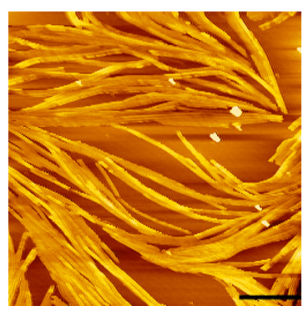

Figure 3. Typical AFM morphological images of the peptide deposited on mica. Peptide dissolved in water at a concentration of $0.5 \mathrm{mg} / \mathrm{mL}$. (A) $2 \times 100$ uL Milli-Q water-diluted solution showing the fiber structure. (B) Higher magnification of (A). The scale bars in the figure represent the following scales: (A) 2 um. (B) $1 \mathrm{um}$.

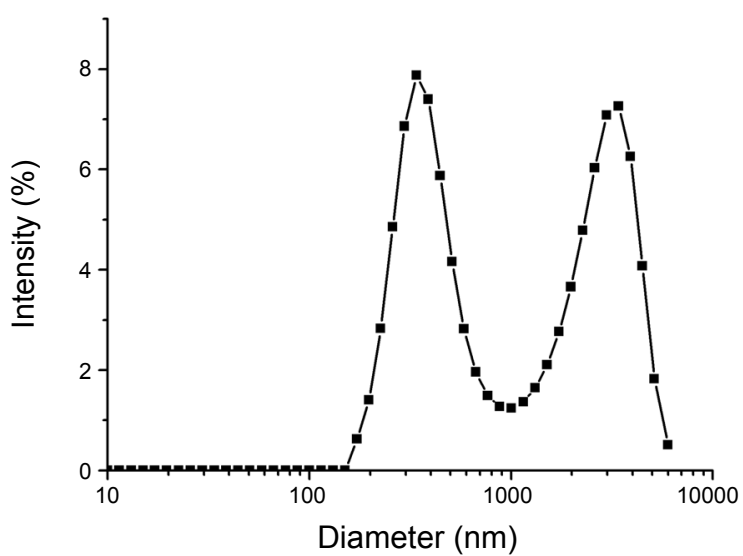

Figure 4. Size distribution of the nanostructure formed by the peptide (concentration $=0.5 \mathrm{mg} / \mathrm{mL}$ )

lego-type peptide formed nanostructures of different sizes in the aqueous solution. We performed the DLS test using the same sample of the peptide solution that was used in the AFM analysis at a concentration of $0.5 \mathrm{mg} / \mathrm{mL}$. As shown in Figure 4, two peaks were exhibited in the spectrum, indicating that the nanostructures formed by the peptide were distributed in two very different ranges. The first distribution was in the range of $100 \mathrm{~nm}$ to almost $1000 \mathrm{~nm}$, whereas the other was in the range of thousands of nanometers. The results of DLS experiments correlated well with the results of AFM. Although the size distribution revealed by DLS was not accurately applied to analyze the size of the nanostructures, it confirmed the existence of the nanofibers in the peptide solution with different fiber lengths.

Investigation of the hydrophobic district of the peptide. We used hydrophobic pyrene as a model to study the hydrophobic district of the peptide. The ratio of the intensities of the first $(373.8 \mathrm{~nm})$ and the third $(384.8 \mathrm{~nm})$ peaks of the pyrene monomer (i.e., I1/I3) was used to determine the polarity of the pyrene environment. ${ }^{23-24}$ It has been reported that the I1/I3 ratio is 1.96 for a polar solvent such as water, and the ratio decreases with the increase of solvent apolarity. As the data indicates (Figure 5), compared to the I1/I3 ratio of pyrene in water, the $\mathrm{I} 1 / \mathrm{I} 3$ ratio of pyrene in the peptide was significantly lower, demonstrating the formation of the hydrophobic district during the self-assembly process. Therefore, we concluded that the formation of the hydrophobic district was crucial in the self-assembly procedure. 


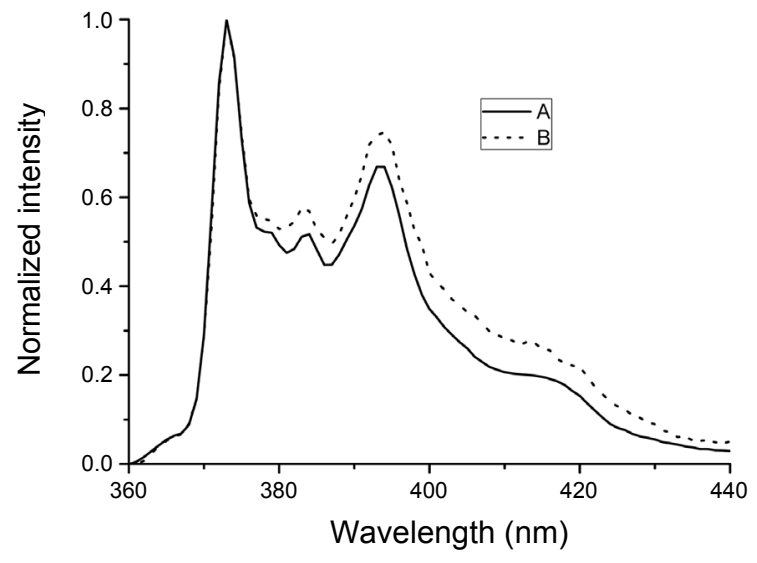

Figure 5. Normalized fluorescence emission spectra of (A) PY mixed with Milli-Q water $\left([\mathrm{Py}]=2.5 \times 10^{-3} \mathrm{M}\right)$ and $(\mathrm{B}) \mathrm{PY}$ mixed with peptide solution $\left([\mathrm{Py}]=2.5 \times 10^{-3} \mathrm{M}\right.$, [Peptide $\left.]=5.1 \times 10^{-4} \mathrm{M}\right)$.

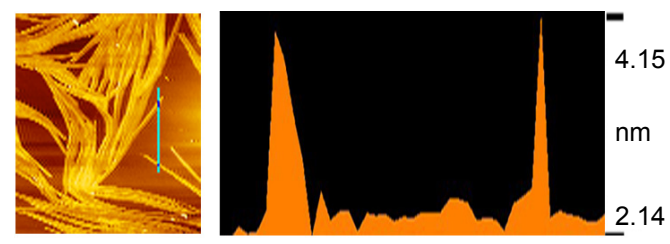

Figure 6. Line profile analysis of the section marked by the blue line.

This result also confirmed that the peptide was able to stabilize a hydrophobic cargo such as py crystals in an aqueous solution. These data indicated that the novel designed peptide was able to act a potential carrier of hydrophobic drugs and may provide a new type of nanomaterial for drug delivery.

The mechanism of the self-assembly process. The proposed self-assembly model of the peptide was required to obtain a thorough understanding of the process. The peptide monomer had two distinct surfaces, both amphiphilic. As the chemical structure and the molecular schematic of the peptide indicated, the positive Glu and negative Lys (as head and tail, respectively) were located on different side of the hydrophobic Ala backbones and form the hydrophilic district. Therefore, we hypothesized that the formation of the peptide fiber involved two stages. In the first stage, the peptide monomers self-assembled into dimmers based on the ionic interactions. As is known, opposite charges can afford complementary ionic bonds, which can greatly stabilize the structure. Line profile analysis showed that the height of the fibers was approximately $2.1 \mathrm{~nm}$, which was approximately twice the width of the monomer (Figure 6). This result further supported the formation of dimmers. The second step involved the association of the dimmers to lengthen the fibers and the nanofibers tapes. In this step, the dimmer units were driven into the nanostructure primarily by hydrophobic interactions and hydrogen bonds. The packing of the hydrophobic sections may have led to the formation of hydrogen bonds between the backbones, which can stabilize the nanostructure. The presence of the double amphiphilic sides may have strengthened the coexistence of the units, facilitated the adhesive attraction and strengthened the density of the fibers to assemble

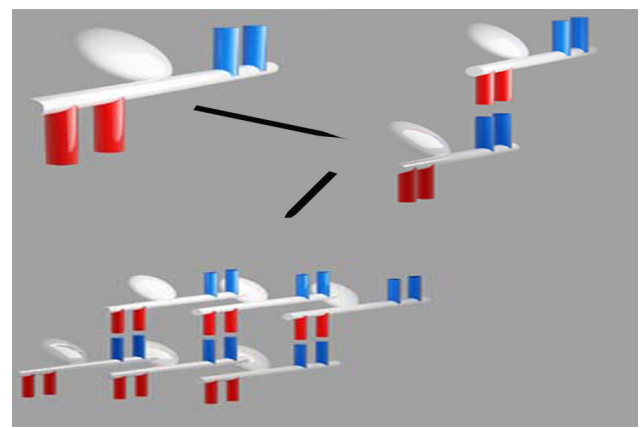

Figure 7. The proposed self-assembly process for the peptide showing an image of the fiber structure, which is connected with ionic bonds, hydrophobic interactions and hydrogen bonds. The peptide forms two distinct surfaces, both amphiphilic. When the hydrophobic sections are packed together, hydrogen bonds may form between the backbones and further stabilize the nanostructure.

into tapes. In summary, the peptides were able to form nanofibers mediated by non-covalent interactions between molecules via ionic bonds, hydrophobic interactions and hydrogen bonds. Figure 7 describes the model for the self-assembly of the peptide.

\section{Conclusion}

In this article, we have described the fabrication of a novel designed peptide that exhibits the characteristics of a random coil. Although our peptide was different from the typical legotype peptide with the secondary beta-sheet structure, the novel peptide was able to form ordered nanostructures in an aqueous solution. The image of nanofiber tapes proved that the new peptide with double amphiphilic surfaces also exhibited self-assembly behavior in both aqueous and traditional solutions. Fluorescence results suggested that this new nanomaterial might be a promising potential carrier for drug delivery applications in soft tissues. Finally, all of the data confirmed our original conception for the designed amphiphilic peptide. The results presented in this manuscript chart a complementary path for the peptide designer.

Acknowledgments. The authors are grateful to the financial support from "Project 985" of the Ministry of Education of the People's Republic of China. We would like to thank Zhihua Xing for help with the technique and suggestive discussions about the manuscript. We would also like to thank Wenjie Si for her original work and help in the preparation of this manuscript.

\section{References}

1. Zhang, S.; Yan, L.; Altman, M.; Lassle, M.; Nugent, H.; Frankel, F.; Lauffenburger, D. A.; Whitesides, G. M.; Rich, A. Biomaterials 1999, 20, 13.

2. Vauthey, S.; Santoso, S.; Gong, H.; Watson, N.; Zhang, S. Proc. Natl. Acad. Sci. USA 2002, 99, 8.

3. Zhang, S. Nature Biotechnology 2003, 21, 10.

4. Zhao, X.; Zhang, S. Trends Biotechnol. 2004, 22, 9. 
5. Ruan, L.; Zhang, H.; Luo, H.; Liu, J.; Tang, F.; Shi, Y.-K.; Zhao, X. Proc. Natl. Acad. Sci. USA 2009, 106, 13.

6. Zhou, Q.; Lin, J.; Wang, J.; Li, F.; Tang, F.; Zhao, X. Progress in Natural Science 2009, 19, 11.

7. Song, H.; Zhang, L.; Zhao, X. Macromol. Biosci. 2010, 10, 1.

8. Zhao, X.; Nagai, Y.; Reeves, P. J.; Kiley, P.; Zhang, L.; Khorana, H. G. Proc. Natl. Acad. Sci. USA 2006, 103, 47.

9. Segers, V. F. M.; Lee, R. T. Drug Discovery Today 2007, 12,

10. Zhang, S.; Lockshin, C.; Herbert, A.; Winter, E.; Rich, A. EMBO J. 1992, 11, 10.

11. Yokoi, H.; Kinoshita, T.; Zhang, S. Proc. Natl. Acad. Sci. USA 2005, 102, 24.

12. Zhang, S.; Gelain, F.; Zhao, X. Semin. Cancer Biol. 2005, 15, 5 .

13. Schneider, A.; Garlick, J. A.; Egles, C. PLoS ONE. 2008, 3, 1.

14. Luo, Z.; Zhao, X.; Zhang, S. Macromol. Biosci. 2008, 8, 8.

15. Ruan, L.-P.; Luo, H.-L.; Zhang, H.-Y.; Zhao, X. Macromolecular
Research 2009, 17, 8 .

16. Orbach, R.; Adler-Abramovich, L.; Zigerson, S.; Mironi-Harpaz, I.; Seliktar, D.; Gazit, E. Biomacromolecules 2009, 10, 9.

17. Fung, S. Y.; Yang, H.; Chen, P. Colloids and Surfaces B: Biointerfaces 2007, 55, 2.

18. Zhang, S.; Zhao, X. Journal of Materials Chemistry 2004, 14, 14.

19. Kelly, S. M.; Jess, T. J.; Price, N. C. Biochimica et Biophysica Acta 2005, 1751, 2.

20. Jackson, M.; Mantsch, H. H. Critical Reviews in Biochemistry and Molecular Biology 1995, 30, 2.

21. Qiu, F.; Chen, Y.; Zhao, X. J. Colloid Interface Sci. 2009, 336, 2.

22. Jalili, N.; Laxminarayana, K. Mechatronics 2004, 14, 8.

23. Keyes-Baig, C.; Duhamel, J.; Fung, S.-Y.; Bezaire, J.; Chen, P. J. Amer. Chem. Soc. 2004, 126, 24.

24. Li, F.; Wang, J.; Tang, F.; Lin, J.; Zhang, Y.; Zhang, E.-Y.; Wei, C.; Shi, Y.-K.; Zhao, X. J. Nanosci. Nanotechnol. 2009, 9, 2. 